\title{
Exact Solutions for the Nonlinear (2+1)-Dimensional Davey-Stewartson Equation Using the Generalized $\left(\frac{G^{\prime}}{G}\right)$-Expansion Method
}

\author{
Mahmoud A. M. Abdelaziz ${ }^{1}$, A. E. Moussa ${ }^{1}$ \& D. M. Alrahal ${ }^{1}$ \\ ${ }^{1}$ College of Business \& Economics, Qassim University, Buraydah, Saudi Arabia \\ Correspondence: Mahmoud A. M. Abdelaziz, College of Business \& Economics, Qassim University, Buraydah, \\ Saudi Arabia. Tel: 966-16-380-0050. E-mail: mamabdelaziz@ hotmail.com
}

\author{
Received: November 3, 2013 Accepted: February 26, 2014 Online Published: May 13, 2014 \\ doi:10.5539/jmr.v6n2p91 URL: http://dx.doi.org/10.5539/jmr.v6n2p91
}

\begin{abstract}
In this article, we construct the exact traveling wave solutions of the nonlinear $(2+1)$-dimensional Davey-Stewartson equation (D-S) using the generalized $\left(\frac{G^{\prime}}{G}\right)$-expansion method which play an important role in mathematical physics. As a result, hyperbolic, trigonometric and rational function solutions with parameters are obtained. When these parameters are taken special values, the solitary and periodic solutions are derived from the hyperbolic and trigonometric function solutions respectively. New complex type traveling wave solutions to the nonlinear $(2+1)-$ dimensional Davey-Stewartson equation were obtained with Liu's theorem.
\end{abstract}

Keywords: exact solutions, Davey-Stewartson equation, generalized $\left(\frac{G^{\prime}}{G}\right)$-expansion method, Liu's theorem, nonlinear PDEs

\section{Introduction}

In the nonlinear science, many important phenomena in various fields can be described by the nonlinear partial differential equations (NLPDEs). Searching and constructing exact solutions for NLPDEs is interesting and important. These exact solutions of these NLPDEs are important for the understanding of the nonlinear physical phenomena and possible applications. In the past several decades, many effective methods for obtaining exact solutions of NLPDEs have been presented, such as the tanh function method (Fan, 2000; El-Wakil et al., 2007), the tanh-sech method (Malfliet et al., 1996; Wazwaz, 2004), the sine-cosine method (Al-Mdallal et al., 2007; Zayed \& Abdelaziz, 2011), the homogeneous balance method (Fan et al., 1998), the Jacobi elliptic function method (Dai et al., 2006), the F-expansion method (Zhang et al., 2006), the homotopy perturbation method (He, 2005), the inverse scattering transformation method (Ablowitz et al., 1981), the Bäcklund transformation method (Miura, 1978), the Hirota bilinear method (Hirota, 1973), the exp-function method (Zhang, 2008; Zayed et al., 2012), the $\left(\frac{G^{\prime}}{G}\right)$-expansion method (Wang et al., 2008; Zayed \& Abdelaziz, 2010, 2013) and so on.

Very recently, Wang et al. (2008) introduced an expansion technique called the $\left(\frac{G^{\prime}}{G}\right)$-expansion method and they demonstrated that it was a powerful technique for seeking analytic solutions of NLPDEs. Later, Zhang et al. (2008) proposed a generalized $\left(\frac{G^{\prime}}{G}\right)$-expansion method to improve and extend Wang et al.'s work (2008) for solving variable-coefficient equations and high dimensional equations. The $\left(\frac{G^{\prime}}{G}\right)$-expansion method is based on the assumptions that the traveling wave solutions can be expressed by a polynomial in $\left(\frac{G^{\prime}}{G}\right)$, where $G$ satisfies the following second order linear ordinary differential equation:

$$
G^{\prime \prime}+\lambda G^{\prime}+\mu G=0
$$

while $\lambda$ and $\mu$ are arbitrary constants.

In this article, we apply the generalized $\left(\frac{G^{\prime}}{G}\right)$-expansion method to improve the work made in (Wang et al., 2008). Zhang et al. (2008) first proposed this method to construct exact solutions of the mKdV equation with variable coefficients. As an application of the suggested method, we will consider the following nonlinear (2+1)-dimensional Davy-Stewartson equation: 


$$
\begin{aligned}
i u_{t}+\frac{1}{2} \sigma^{2}\left(u_{x x}+\sigma^{2} u_{y y}\right)+\delta|u|^{2} u-v_{x} u & =0, \\
v_{x x}-\sigma^{2} v_{y y}-2 \delta\left(|u|^{2}\right)_{x} & =0,
\end{aligned}
$$

where $\delta= \pm 1$ and $\sigma^{2}= \pm 1$. The case $\sigma=1$ is called the DS-I equation, while $\sigma=i$ is the DS-II equation. The parameter $\delta$ characterizes the focusing or defocusing case. The DS equation has four kinds of soliton solutions: the conventional line, algebraic, periodic and lattice solutions: the conventional line soliton has an essentially one-dimensional structure. On the other hand, the algebraic, periodic and lattice solitons have a two-dimensional structure. The Davey-Stewartson I and II are two well-known examples of integrable equations in two space dimensions, which arise as higher dimensional generalizations of the nonlinear Schrödinger equation (NLSE). They appear in many applications, for example in the description of gravity-capillarity surface wave packets in the limit of the shallow water. Therefore it is of interests to derive explicit solutions of the DS equation. Up to now, many powerful methods have been established and developed to obtain analytic solutions of Equation (2), such as the homotopy analysis method, the sine-cosine method and the variational iteration method (Davey et al., 1974; Zedan et al., 2010; Jafari et al., 2012).

\section{Description of the Generalized $\left(\frac{G^{\prime}}{G}\right)$-Expansion Method}

Suppose that we have a nonlinear PDE in the following form:

$$
F\left(u, u_{t}, u_{x}, u_{y}, u_{t t}, u_{x t}, u_{y t}, u_{x x}, \ldots .\right)=0,
$$

where $u=u(x, y, t)$ is an unknown function, $F$ is a polynomial in $u=u(x, y, t)$ and its partial derivatives, in which the highest order derivatives and nonlinear terms are involved. Let us now give the main steps for solving Equation (3) using the generalized $\left(\frac{G^{\prime}}{G}\right)$-expansion method.

Step 1. The traveling wave variable

$$
u(x, y, t)=u(\xi), \quad \xi=x+y-c t,
$$

where $c$ is a constant, permits us reducing Equation (3) to an ODE for $u=u(\xi)$ in the form

$$
P\left(u, u^{\prime}, u^{\prime \prime}, \ldots .\right)=0,
$$

where $P$ is a polynomial of $u=u(\xi)$ and its total derivatives.

Step 2. Suppose that the solution of Equation (5) can be expressed by a polynomial in $\left(\frac{G^{\prime}}{G}\right)$ as follows:

$$
u(\xi)=\sum_{i=0}^{m} \alpha_{i}\left(\frac{G^{\prime}(\xi)}{G(\xi)}\right)^{i},
$$

where $\alpha_{i}$ are constants to be determined later, $\alpha_{m} \neq 0$ and $m$ is a positive integer.

Step 3. Balancing the highest derivative term with the nonlinear terms in Equation (5), we find the value of the positive integer $m$ in Equation (6).

Step 4. Substituting Equation (6) into Equation (5) and using Equation (1), collecting all terms with the same power of $\left(\frac{G^{\prime}}{G}\right)$ together, and then equating each coefficient of the resulted polynomial to zero, yields a set of algebraic equations for $\alpha_{i}, c, \lambda$ and $\mu$.

Step 5. Since the general solutions of Equation (1) have been well known for us, then substituting $\alpha_{i}, c$ and the general solutions of Equation (1) into Equation (6), we have the traveling wave solutions of the nonlinear PDEs (3).

Theorem 1 (Liu's Theorem) If Equation (3) has a kink-type solution

$$
u=p_{k}\left(\tanh \left[A\left(\xi+\xi_{0}\right)\right]\right),
$$

then it has certain the kink-bell-type solution

$$
u=p_{k}\left(\tanh \left[2 A\left(\xi+\xi_{0}\right)\right] \pm i \operatorname{sech}\left[2 \mathrm{~A}\left(\xi+\xi_{0}\right)\right]\right),
$$


where $p_{k}$ is a polynomial of degree $k, i$ is the imaginary number unit.

\section{An Application}

In this section, we will apply the generalized $\left(\frac{G^{\prime}}{G}\right)$-expansion method to construct the exact solutions of the nonlinear (2+1)-Davey Stewartson equation. To this end, we take the following transformations of Equation (2)

$$
\begin{array}{cc}
u(x, y, t)=U(\xi) e^{i \theta}, & v(x, y, t)=V(\xi), \\
\xi=x+y-c t, & \theta=l_{1} x+l_{2} y+l_{3} t,
\end{array}
$$

where $c, l_{1}, l_{2}$ and $l_{3}$ are real constants. Substituting (9) and (10) into (2) and separating the real and imaginary parts we obtain

$$
\begin{gathered}
c=\sigma^{2}\left(l_{1}+\sigma^{2} l_{2}\right), \\
\sigma^{2}\left(1+\sigma^{2}\right) U^{\prime \prime}(\xi)+2 \delta U^{3}(\xi)-\left[2\left(V^{\prime}(\xi)+l_{3}\right)+\sigma^{2}\left(l_{1}^{2}+\sigma^{2} l_{2}^{2}\right)\right] U(\xi)=0, \\
\left(1-\sigma^{2}\right) V^{\prime \prime}(\xi)-2 \delta\left(U^{2}(\xi)\right)^{\prime}=0 .
\end{gathered}
$$

Integrating (13) with respect to $\xi$ and setting the constant of integration to zero, we find

$$
V^{\prime}(\xi)=\frac{2 \delta}{1-\sigma^{2}} U^{2}(\xi)
$$

Substituting (14) into (12) yields

$$
\sigma^{2}\left(\sigma^{4}-1\right) U^{\prime \prime}(\xi)+2 \delta\left(\sigma^{2}+1\right) U^{3}(\xi)-\left(\sigma^{2}-1\right)\left[2 l_{3}+\sigma^{2}\left(l_{1}^{2}+\sigma^{2} l_{2}^{2}\right)\right] U(\xi)=0 .
$$

Balancing $U^{\prime \prime}$ and $U^{3}$ in Equation (15), we have $m=1$. Consequently, we have the formal solution of Equation (15) in the form

$$
U(\xi)=\alpha_{1}\left(\frac{G^{\prime}(\xi)}{G(\xi)}\right)+\alpha_{0},
$$

where $\alpha_{1}, \alpha_{0}$ are constants to be determined later. After some calculation we can obtain

$$
\begin{gathered}
U^{\prime \prime}(\xi)=\alpha_{1}\left[2\left(\frac{G^{\prime}(\xi)}{G(\xi)}\right)^{3}+3 \lambda\left(\frac{G^{\prime}(\xi)}{G(\xi)}\right)^{2}+\left(\lambda^{2}+2 \mu\right)\left(\frac{G^{\prime}(\xi)}{G(\xi)}\right)+\lambda \mu\right], \\
U^{3}(\xi)=\alpha_{1}^{3}\left(\frac{G^{\prime}(\xi)}{G(\xi)}\right)^{3}+3 \alpha_{1}^{2} \alpha_{0}\left(\frac{G^{\prime}(\xi)}{G(\xi)}\right)^{2}+3 \alpha_{1} \alpha_{0}^{2}\left(\frac{G^{\prime}(\xi)}{G(\xi)}\right)+\alpha_{0}^{3} .
\end{gathered}
$$

Substituting (16)-(18) into (15), collecting all terms with the same powers of $\left(\frac{G^{\prime}}{G}\right)$ and setting them to zero then, we have the following system of algebraic equations:

$$
\begin{aligned}
\left(\frac{G^{\prime}}{G}\right)^{3}: & 2 \sigma^{2}\left(\sigma^{4}-1\right) \alpha_{1}+2 \delta\left(\sigma^{2}+1\right) \alpha_{1}^{3}=0 \\
\left(\frac{G^{\prime}}{G}\right)^{2}: & 3 \sigma^{2}\left(\sigma^{4}-1\right) \lambda \alpha_{1}+6 \delta\left(\sigma^{2}+1\right) \alpha_{0} \alpha_{1}^{2}=0 \\
\left(\frac{G^{\prime}}{G}\right)^{1}: & \sigma^{2}\left(\sigma^{4}-1\right) \lambda^{2} \alpha_{1}+2 \sigma^{2}\left(\sigma^{4}-1\right) \mu \alpha_{1}+6 \delta\left(\sigma^{2}+1\right) \alpha_{0}^{2} \alpha_{1} \\
& -\left(\sigma^{2}-1\right)\left[2 l_{3}+\sigma^{2}\left(l_{1}^{2}+\sigma^{2} l_{2}^{2}\right)\right] \alpha_{1}=0, \\
\left(\frac{G^{\prime}}{G}\right)^{0}: \quad & \sigma^{2}\left(\sigma^{4}-1\right) \mu \lambda \alpha_{1}+2 \delta\left(\sigma^{2}+1\right) \alpha_{0}^{3} \\
& -\left(\sigma^{2}-1\right)\left[2 l_{3}+\sigma^{2}\left(l_{1}^{2}+\sigma^{2} l_{2}^{2}\right)\right] \alpha_{0}=0 .
\end{aligned}
$$

Solving the system (19) by the Maple, we have the following solution:

$$
\begin{aligned}
& \alpha_{1}= \pm \sigma \sqrt{\frac{1-\sigma^{2}}{\delta}}, \quad \alpha_{0}= \pm \frac{\sigma \lambda}{2} \sqrt{\frac{1-\sigma^{2}}{\delta}}, \quad l_{1}=l_{1}, \quad l_{2}=l_{2}, \\
& l_{3}=\frac{-\sigma^{2}}{4}\left[2\left(l_{1}^{2}+\sigma^{2} l_{2}^{2}\right)+\left(\sigma^{2}+1\right)\left(\lambda^{2}-4 \mu\right)\right] .
\end{aligned}
$$


Substituting (20) into (16), we have

$$
U(\xi)= \pm \frac{\sigma}{\delta} \sqrt{\delta\left(1-\sigma^{2}\right)}\left[\frac{\lambda}{2}+\frac{G^{\prime}(\xi)}{G(\xi)}\right] .
$$

In view of (9)-(11), (14) and (21), we obtain the general solutions of Equation (2) in the forms

$$
\begin{gathered}
u(\xi)= \pm \frac{\sigma}{\delta} \sqrt{\delta\left(1-\sigma^{2}\right)}\left[\frac{\lambda}{2}+\frac{G^{\prime}(\xi)}{G(\xi)}\right] \times \exp \left\{i\left[l_{1} x+l_{2} y-\frac{\sigma^{2}}{4}\left(2\left(l_{1}^{2}+\sigma^{2} l_{2}^{2}\right)+\left(\sigma^{2}+1\right)\left(\lambda^{2}-4 \mu\right)\right) t\right]\right\} \\
v(\xi)=2 \sigma^{2} \int\left[\frac{\lambda}{2}+\frac{G^{\prime}(\xi)}{G(\xi)}\right]^{2} d \xi
\end{gathered}
$$

where

$$
\xi=x+y-\sigma^{2}\left(l_{1}+\sigma^{2} l_{2}\right) t
$$

Consequently, we have the following three types of exact solutions of Equation (2).

Case 1: When $\lambda^{2}-4 \mu>0$, we obtain the hyperbolic function solutions in the forms

$$
\begin{gathered}
u(\xi)= \pm \frac{\sigma}{2 \delta} \sqrt{\delta\left(1-\sigma^{2}\right)\left(\lambda^{2}-4 \mu\right)}\left[\frac{c_{1} \cosh \left(\frac{1}{2} \sqrt{\lambda^{2}-4 \mu} \xi\right)+c_{2} \sinh \left(\frac{1}{2} \sqrt{\lambda^{2}-4 \mu} \xi\right)}{c_{1} \sinh \left(\frac{1}{2} \sqrt{\lambda^{2}-4 \mu} \xi\right)+c_{2} \cosh \left(\frac{1}{2} \sqrt{\lambda^{2}-4 \mu} \xi\right)}\right] \times \\
\exp \left\{i\left[l_{1} x+l_{2} y-\frac{\sigma^{2}}{4}\left(2\left(l_{1}^{2}+\sigma^{2} l_{2}^{2}\right)+\left(\sigma^{2}+1\right)\left(\lambda^{2}-4 \mu\right)\right) t\right]\right\} \\
v(\xi)=\frac{\sigma^{2}\left(\lambda^{2}-4 \mu\right)}{2} \int\left(\frac{c_{1} \cosh \left(\frac{1}{2} \sqrt{\lambda^{2}-4 \mu} \xi\right)+c_{2} \sinh \left(\frac{1}{2} \sqrt{\lambda^{2}-4 \mu} \xi\right)}{c_{1} \sinh \left(\frac{1}{2} \sqrt{\lambda^{2}-4 \mu} \xi\right)+c_{2} \cosh \left(\frac{1}{2} \sqrt{\lambda^{2}-4 \mu} \xi\right)}\right)^{2} d \xi .
\end{gathered}
$$

Case 2: When $\lambda^{2}-4 \mu<0$, we have the trigonometric function solutions in the forms

$$
\begin{gathered}
u(\xi)= \pm \frac{\sigma}{2 \delta} \sqrt{\delta\left(1-\sigma^{2}\right)\left(4 \mu-\lambda^{2}\right)}\left[\frac{-c_{1} \sin \left(\frac{1}{2} \sqrt{4 \mu-\lambda^{2}} \xi\right)+c_{2} \cos \left(\frac{1}{2} \sqrt{4 \mu-\lambda^{2}} \xi\right)}{c_{1} \cos \left(\frac{1}{2} \sqrt{4 \mu-\lambda^{2}} \xi\right)+c_{2} \sin \left(\frac{1}{2} \sqrt{4 \mu-\lambda^{2}} \xi\right)}\right] \times \\
\exp \left\{i\left[l_{1} x+l_{2} y-\frac{\sigma^{2}}{4}\left(2\left(l_{1}^{2}+\sigma^{2} l_{2}^{2}\right)-\left(\sigma^{2}+1\right)\left(4 \mu-\lambda^{2}\right)\right) t\right]\right\}, \\
v(\xi)=\frac{\sigma^{2}\left(4 \mu-\lambda^{2}\right)}{2} \int\left(\frac{-c_{1} \sin \left(\frac{1}{2} \sqrt{4 \mu-\lambda^{2}} \xi\right)+c_{2} \cos \left(\frac{1}{2} \sqrt{4 \mu-\lambda^{2}} \xi\right)}{c_{1} \cos \left(\frac{1}{2} \sqrt{4 \mu-\lambda^{2}} \xi\right)+c_{2} \sin \left(\frac{1}{2} \sqrt{4 \mu-\lambda^{2}} \xi\right)}\right)^{2} d \xi .
\end{gathered}
$$

Case 3: When $\lambda^{2}-4 \mu=0$, we get the rational function solutions in the forms

$$
\begin{gathered}
u(\xi)= \pm \frac{c_{2} \sigma \sqrt{\delta\left(1-\sigma^{2}\right)}}{\delta\left(c_{1}+c_{2} \xi\right)} \exp \left\{i\left[l_{1} x+l_{2} y-\frac{\sigma^{2}}{4}\left(l_{1}^{2}+\sigma^{2} l_{2}^{2}\right) t\right]\right\} \\
v(\xi)=\frac{-2 c_{2} \sigma^{2}}{c_{1}+c_{2} \xi}+d_{0},
\end{gathered}
$$

where $c_{1}, c_{2}$ are arbitrary constants and $d_{0}$ is a constant of integration. Finally, we note that:

(1) If $\mu=0, \lambda>0, c_{2}=0$ and $c_{1} \neq 0$ then we deduce from (25) and (26) that

$$
\begin{gathered}
u(\xi)= \pm \frac{\sigma \lambda}{2 \delta} \sqrt{\delta\left(1-\sigma^{2}\right)} \operatorname{coth}\left(\frac{\lambda \xi}{2}\right) \times \exp \left\{i\left[l_{1} x+l_{2} y-\frac{\sigma^{2}}{4}\left(2\left(l_{1}^{2}+\sigma^{2} l_{2}^{2}\right)+\lambda^{2}\left(\sigma^{2}+1\right)\right) t\right]\right\}, \\
v(\xi)=\frac{\sigma^{2} \lambda}{2}\left[\lambda \xi-2 \operatorname{coth}\left(\frac{\lambda \xi}{2}\right)\right]+d_{1},
\end{gathered}
$$

and therefore from Theorem 2.1 we also have

$$
\begin{aligned}
u(\xi)= & \pm \frac{\sigma \lambda}{2 \delta} \sqrt{\delta\left(1-\sigma^{2}\right)}[\tanh (\lambda \xi) \pm i \operatorname{sech}(\lambda \xi)]^{-1} \times \\
& \exp \left\{i\left[l_{1} x+l_{2} y-\frac{\sigma^{2}}{4}\left(2\left(l_{1}^{2}+\sigma^{2} l_{2}^{2}\right)+\lambda^{2}\left(\sigma^{2}+1\right)\right) t\right]\right\}
\end{aligned}
$$




$$
v(\xi)=\frac{\sigma^{2} \lambda}{2}\left\{\lambda \xi-2[\tanh (\lambda \xi) \pm i \operatorname{sech}(\lambda \xi)]^{-1}\right\}+d_{1},
$$

where $d_{1}$ is a constant of integration, while if $\mu=0, \lambda>0, c_{2} \neq 0$ and $c_{2}^{2}>c_{1}^{2}$ we deduce from (25) and (26) that

$$
\begin{gathered}
u(\xi)= \pm \frac{\sigma \lambda}{2 \delta} \sqrt{\delta\left(1-\sigma^{2}\right)} \tanh \left(\xi_{1}+\frac{\lambda \xi}{2}\right) \times \exp \left\{i\left[l_{1} x+l_{2} y-\frac{\sigma^{2}}{4}\left(2\left(l_{1}^{2}+\sigma^{2} l_{2}^{2}\right)+\lambda^{2}\left(\sigma^{2}+1\right)\right) t\right]\right\}, \\
v(\xi)=\frac{\sigma^{2} \lambda}{2}\left[\lambda \xi-2 \tanh \left(\xi_{1}+\frac{\lambda \xi}{2}\right)\right]+d_{2},
\end{gathered}
$$

and therefore from Theorem 2.1 we also have

$$
\begin{aligned}
u(\xi)= & \pm \frac{\sigma \lambda}{2 \delta} \sqrt{\delta\left(1-\sigma^{2}\right)}\left[\tanh \left(2 \xi_{1}+\lambda \xi\right) \pm i \operatorname{sech}\left(2 \xi_{1}+\lambda \xi\right)\right] \times \\
& \exp \left\{i\left[l_{1} x+l_{2} y-\frac{\sigma^{2}}{4}\left(2\left(l_{1}^{2}+\sigma^{2} l_{2}^{2}\right)+\lambda^{2}\left(\sigma^{2}+1\right)\right) t\right]\right\}, \\
v(\xi)= & \frac{\sigma^{2} \lambda}{2}\left\{\lambda \xi-2\left[\tanh \left(2 \xi_{1}+\lambda \xi\right) \pm i \operatorname{sech}\left(2 \xi_{1}+\lambda \xi\right)\right]\right\}+d_{2},
\end{aligned}
$$

where $\xi_{1}=\tanh ^{-1}\left(\frac{c_{1}}{c_{2}}\right)$ and $d_{2}$ is a constant of integration.

(2) If $c_{1}^{2}+c_{2}^{2} \neq 0$, then we deduce from (27) and (28) that

$$
\begin{aligned}
u(\xi)= & \pm \frac{\sigma}{2 \delta} \sqrt{\delta\left(1-\sigma^{2}\right)\left(4 \mu-\lambda^{2}\right)} \tan \left(\xi_{2}-\frac{1}{2} \sqrt{4 \mu-\lambda^{2}} \xi\right) \times \\
& \exp \left\{i\left[l_{1} x+l_{2} y-\frac{\sigma^{2}}{4}\left(2\left(l_{1}^{2}+\sigma^{2} l_{2}^{2}\right)-\left(\sigma^{2}+1\right)\left(4 \mu-\lambda^{2}\right)\right) t\right]\right\}, \\
v(\xi)=- & \frac{1}{2} \sigma^{2} \sqrt{4 \mu-\lambda^{2}}\left[\sqrt{4 \mu-\lambda^{2}} \xi+2 \tan \left(\xi_{2}-\frac{1}{2} \sqrt{4 \mu-\lambda^{2}} \xi\right)\right]+d_{3},
\end{aligned}
$$

where $\xi_{2}=\tan ^{-1}\left(\frac{c_{2}}{c_{1}}\right)$ and $d_{3}$ is a constant of integration, while

$$
\begin{aligned}
u(\xi)= & \pm \frac{\sigma}{2 \delta} \sqrt{\delta\left(1-\sigma^{2}\right)\left(4 \mu-\lambda^{2}\right)} \cot \left(\xi_{3}+\frac{1}{2} \sqrt{4 \mu-\lambda^{2}} \xi\right) \times \\
& \exp \left\{i\left[l_{1} x+l_{2} y-\frac{\sigma^{2}}{4}\left(2\left(l_{1}^{2}+\sigma^{2} l_{2}^{2}\right)-\left(\sigma^{2}+1\right)\left(4 \mu-\lambda^{2}\right)\right) t\right]\right\}, \\
v(\xi)=- & \frac{1}{2} \sigma^{2} \sqrt{4 \mu-\lambda^{2}}\left[\sqrt{4 \mu-\lambda^{2}} \xi+2 \cot \left(\xi_{3}+\frac{1}{2} \sqrt{4 \mu-\lambda^{2}} \xi\right)\right]+d_{4},
\end{aligned}
$$

where $\xi_{3}=\cot ^{-1}\left(\frac{c_{2}}{c_{1}}\right)$ and $d_{4}$ is a constant of integration.

Remark 1 Note that (31)-(38) represent the solitary solutions while (39)-(42) represent the periodic solutions of the Davey-Stewartson Equation (2) and the traveling wave solutions obtained using the generalized $\left(\frac{G^{\prime}}{G}\right)$-expansion method for the hyperbolic, trigonometric function types are presented in Equations (31), (32), (35), (36) and (39)(42).

Remark 2 Applying Liu's theorem to Equations (31), (32), (35) and (36), we obtain new complex traveling wave solutions in the form of Equations (33), (34), (37) and (38).

Remark 3 All solutions of this article have been checked with Maple by putting them back into the original Equation (2).

\section{Conclusions}

In this article, the generalized $\left(\frac{G^{\prime}}{G}\right)$-expansion method has been applied to find the exact traveling wave solutions of the nonlinear (2+1)-dimensional Davey-Stewartson equation. Liu's theorem was also applied to obtain new complex traveling wave solutions to the nonlinear $(2+1)$-dimensional Davey-Stewartson equation. As a result, hyperbolic function solutions and trigonometric function solutions with parameters are obtained, from which some known solutions, including the kink-type and kink-bell-type solitary wave solutions are recovered by setting the parameters as special values. These obtained solutions with free parameters may be important to explain some physical phenomena. The paper shows that the generalized $\left(\frac{G^{\prime}}{G}\right)$-expansion method is effective and can be used for many other NLDDEs in mathematical physics. 


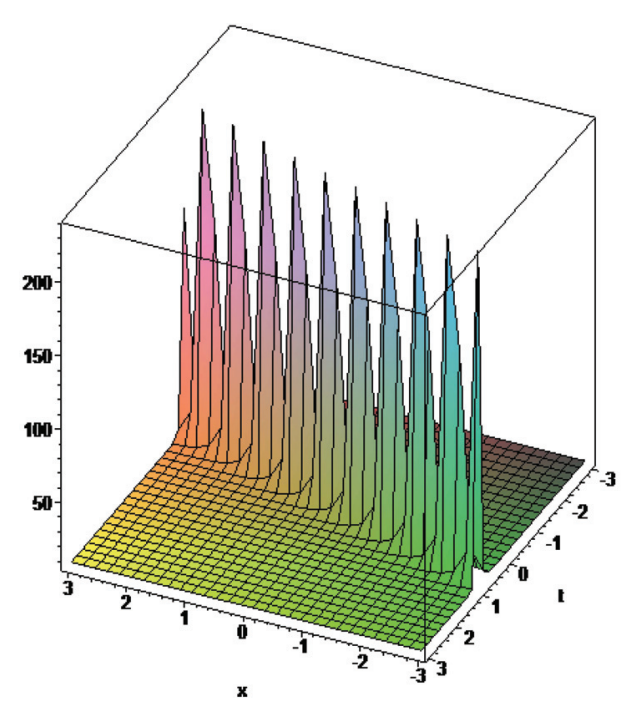

(a)

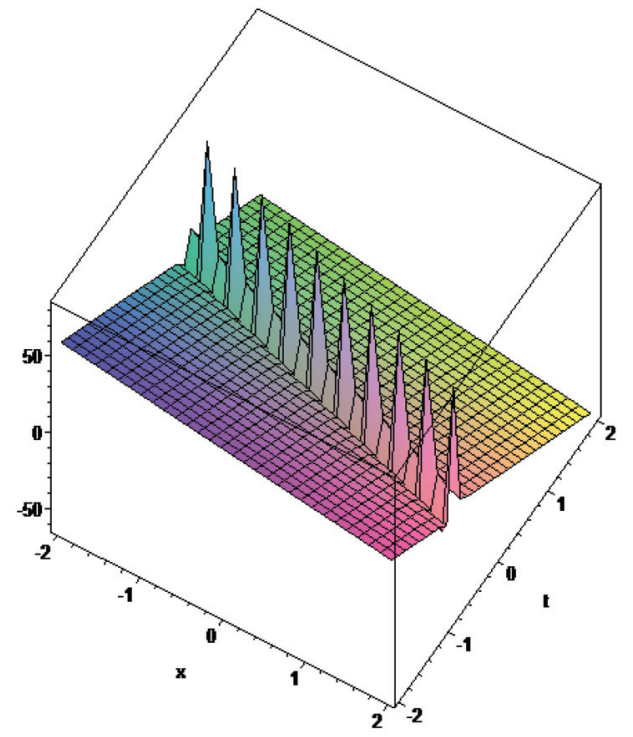

(b)

Figure 1. (a) illustrates the soliton solution for $|u|$ in Equation (31), for $\sigma=I, \delta=-1, l_{1}=2, l_{2}=-1, y=0.3$ and $\lambda=4$. (b) illustrates the soliton solution for $v$ in Equation (32), for $\sigma=I, \delta=-1, l_{1}=2, l_{2}=-1, y=0.3$ and $\lambda=4$

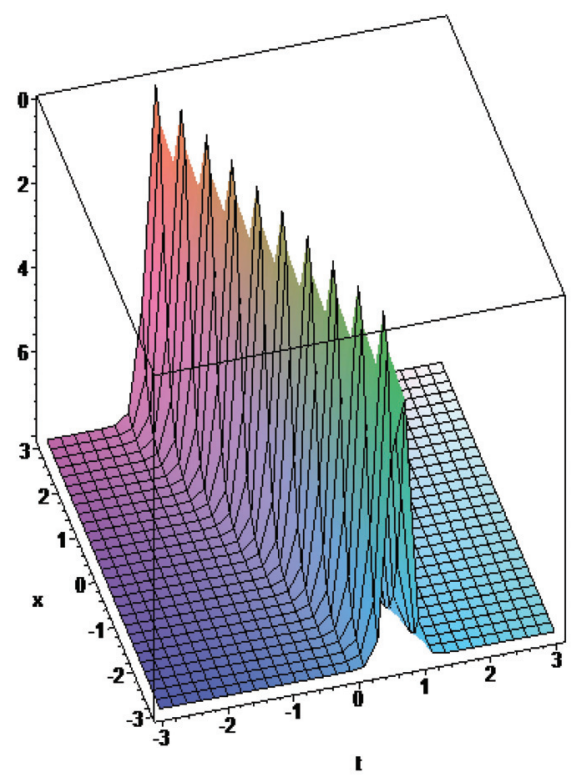

(a)

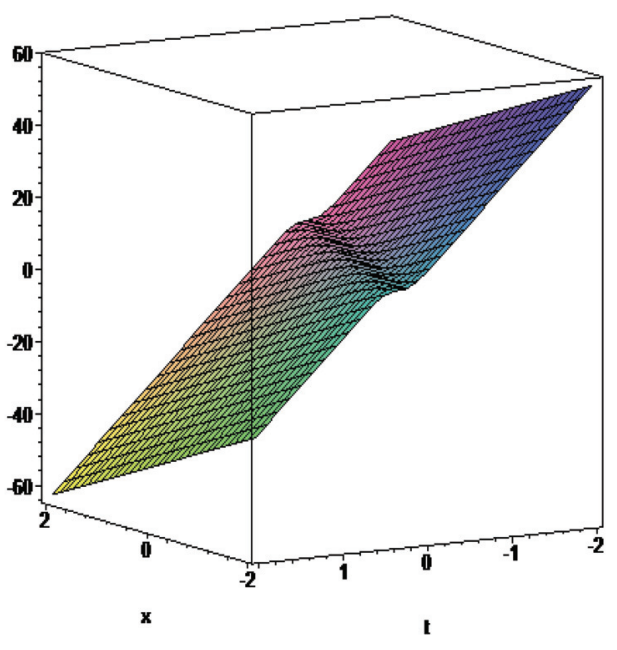

(b)

Figure 2. (a) illustrates the soliton solution for $|u|$ in Equation (35), for $\sigma=I, \delta=-1, l_{1}=2, l_{2}=-1, y=0.3$, $\lambda=4$ and $\xi_{1}=\frac{\pi}{2}$. (b) illustrates the soliton solution for $v$ in Equation (36), for $\sigma=I, \delta=-1, l_{1}=2, l_{2}=-1$, $y=0.3, \lambda=4$ and $\xi_{1}=\frac{\pi}{2}$ 


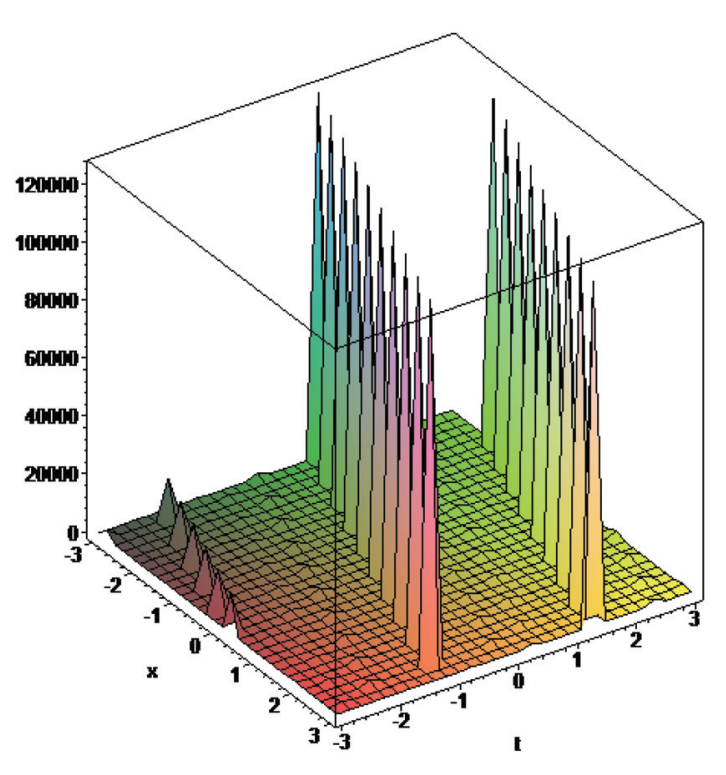

(a)

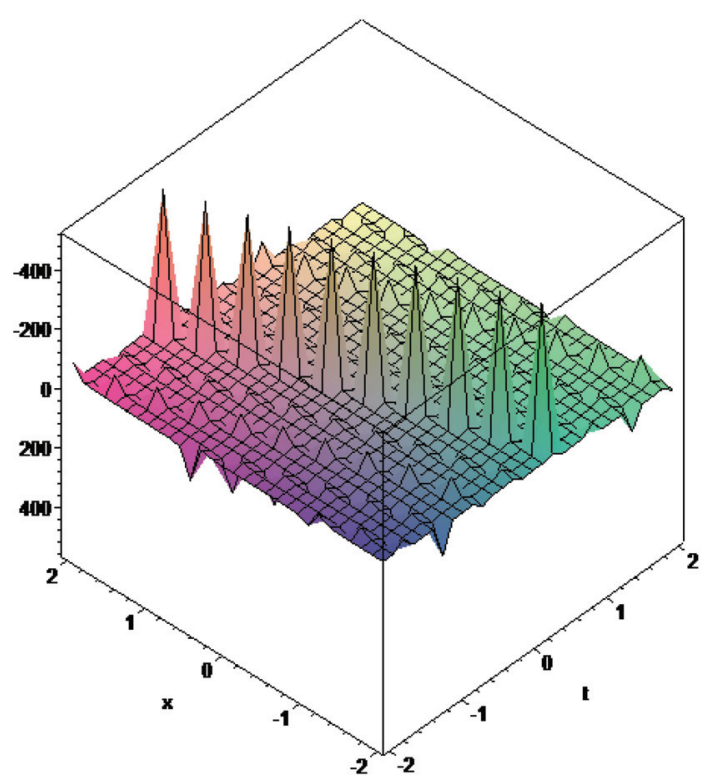

(b)

Figure 3. (a) illustrates the periodic solution for $|u|$ in Equation (39), for $\sigma=I, \delta=-1, l_{1}=2, l_{2}=-1, y=0.3$, $\lambda=4, \mu=8$ and $\xi_{1}=\frac{\pi}{6}$. (b) illustrates the periodic solution for $v$ in Equation (40), for $\sigma=I, \delta=-1, l_{1}=2$, $l_{2}=-1, y=0.3, \lambda=4, \mu=8$ and $\xi_{1}=\frac{\pi}{6}$.

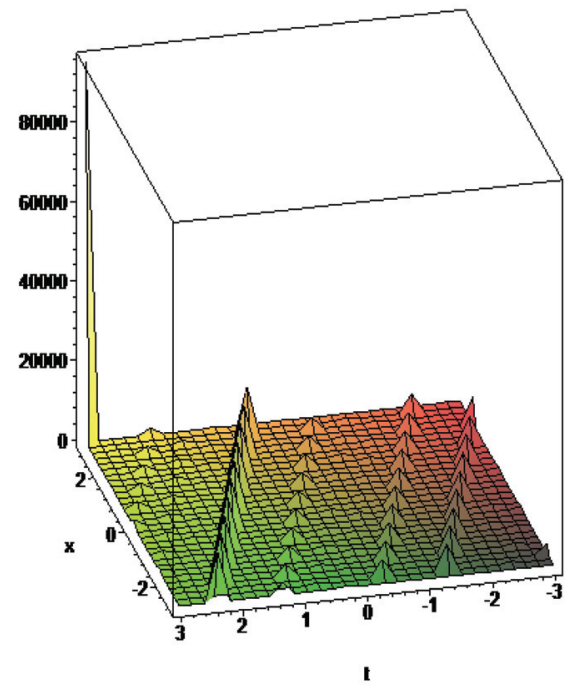

(a)

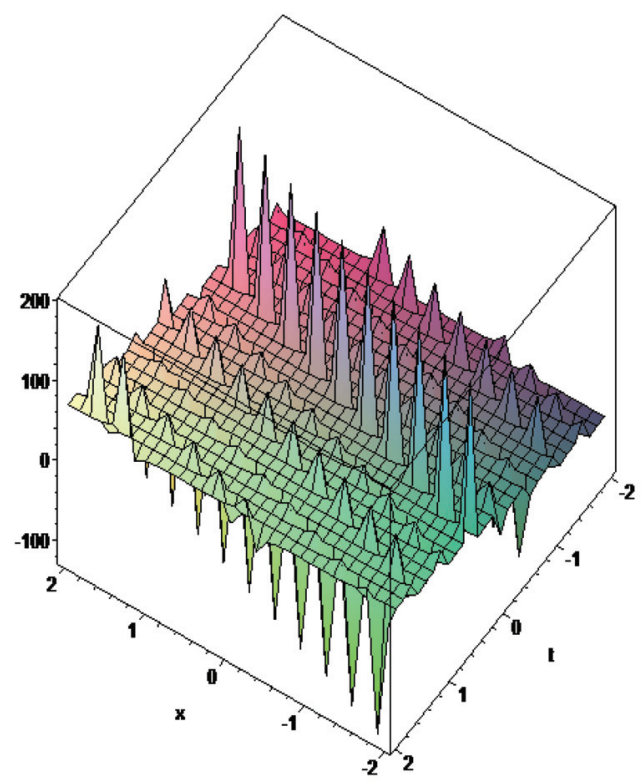

(b)

Figure 4. (a) illustrates the periodic solution for $|u|$ in Equation (41), for $\sigma=I, \delta=-1, l_{1}=2, l_{2}=-1, y=0.3$, $\lambda=4, \mu=8$ and $\xi_{1}=\frac{\pi}{6}$. (b) illustrates the periodic solution for $v$ in Equation (42), for $\sigma=I, \delta=-1, l_{1}=2$, $l_{2}=-1, y=0.3, \lambda=4, \mu=8$ and $\xi_{1}=\frac{\pi}{6}$.

\section{Acknowledgements}

Authors are grateful to referees whose constructive comments helped to improve the paper.

\section{References}

Ablowitz, M. J., \& Segur, H. (1981). Solitons and the inverse scattering transform. Philadelphia, SIAM. 
Al-Mdallal, Q. M. (2008). A new family of exact solutions to the unsteady Navier-Stokes equations using canonical transformation with complex coefficients. Applied Mathematics and Computation, 196(1), 303-308. http://dx.doi.org/10.1016/j.amc.2007.05.054

Dai, C. Q., \& Zhang, J. F. (2006). Jacobian elliptic function method for nonlinear differential-difference equations. Chaos, Solitons \& Fractals, 27, 1042-1047.

Davey, A., \& Stewartson, K. (1974). On three-dimensional packets of surface waves. Proc. Royal. Soc. Lond. Ser. A, 338, 101-110. http://dx.doi.org/10.1098/rspa.1974.0076

El-Wakil, S. A., \& Abdou, M. A. (2007). New exact traveling wave solutions using modified extended tanhfunction method. Chaos, Solitons \& Fractals, 31, 840-852. http://dx.doi.org/10.1016/j.chaos.2005.10.032

Fan, E. (2000). Extended tanh-function method, and its applications to nonlinear equations. Phys. Lett., A, 277, 212-218. http://dx.doi.org/10.1016/S0375-9601(00)00725-8

Fan, E. \& Zhang, H. (1998). A note on the homogeneous balance method. Phys. Lett., A, 246, $403-406$. http://dx.doi.org/10.1016/S0375-9601(98)00547-7

He, J. H. (2005). Homotopy Perturbation Method for Bifurcation of Nonlinear Problems, Int. J. Nonlinear Sci. Numer. Simul., 6, 207-208. http://dx.doi.org/10.1515/IJNSNS.2005.6.2.207

Hirota, R. (1973). Exact N-soliton solutions of the wave equation of long waves in shallow-water and in nonlinear lattices. J. Math. Phys., 14, 810-816. http://dx.doi.org/10.1063/1.1666400

Jafari, H., Kadem, A., Baleanu, D., \& Yilmaz, T. (2012). Solutions of the fractional Davey-Stewartson equations with variational iteration method. Romanian Reports in Physics, 64, 337-346.

Liu, C. P. (2003). The relation between the kink-type solution and the kink-bell-type solution of nonlinear evolution equation. Phys. Lett., A, 312(1-2), 41-48. http://dx.doi.org/10.1016/S0375-9601(03)00572-3

Malfliet, W., \& Hereman, W. (1996). The tanh-method part I. Exact solutions of nonlinear evolution and wave equations. Phys. Script., 54, 563-568. http://dx.doi.org/10.1088/0031-8949/54/6/003

Miura, M. R. (1978). Bäcklund Transformation. Berlin: Springer-Verlag.

Wang, M. L. (1996). Exact solutions of a compound Kdv-Burgers equations. Phys. Lett., A, 213, $279-287$. http://dx.doi.org/10.1016/0375-9601(96)00103-X

Wang, M. L., Li, X. Z., \& Zhang, J. L. (2008). The ( $\left.\frac{G^{\prime}}{G}\right)$-expansion method and traveling wave solutions of nonlinear evolution equations in mathematical physics. Phys. Lett., A, 372, 417-423. http://dx.doi.org/10.1016/j.physleta.2007.07.051

Wazwaz, A. M. (2004). The tanh-method for traveling wave solutions of nonlinear equations. Appl. Math. Comput., 154, 713-723. http://dx.doi.org/10.1016/S0096-3003(03)00745-8

Zayed, E. M. E., \& Abdelaziz, M. A. M. (2010). Exact solutions for the generalized Zakharov-Kuznetsov equation with variable coefficients using the generalized $\left(\frac{G^{\prime}}{G}\right)$-expansion method. AIP Conf. Proc. Amer. Institute of Phys., 1281, 2216-2219. http://dx.doi.org/10.1063/1.3498415

Zayed, E. M. E., \& Abdelaziz, M. A. M. (2011). The modified sine-cosine method and its applications to the generalized $K(n, n)$ and BBM equations with variable coefficients. International Journal of Nonlinear Science, 12(1), 95-99.

Zayed, E. M. E., \& Abdelaziz, M. A. M. (2013). Exact traveling wave solutions of nonlinear variable coefficients evolution equations with forced terms using the generalized $\left(\frac{G^{\prime}}{G}\right)$-expansion method. Computational Mathematics and Modeling, 24(1), 103-113. http://dx.doi.org/10.1007/s10598-013-9163-4

Zedan, H. A., \& Monaquel, S. J. (2010). The sine-cosine method for the Davey-Stewartson equations. Applied. Math. E-Notes, 10, 103-111.

Zhang, J. L., Wang, M. L., Wang, Y. M., \& Fang, Z. D. (2006). The improved F-expansion method and its applications. Phys. Lett., A, 350, 103-109. http://dx.doi.org/10.1016/j.physleta.2005.10.099

Zhang, S., Tong, J. L., \& Wang, W. (2008). A generalized $\left(\frac{G^{\prime}}{G}\right)$-expansion method for the mKdV equation with variable coefficients. Phys. Lett., A, 372, 2254-2257. http://dx.doi.org/10.1016/j.physleta.2007.11.026 


\section{Copyrights}

Copyright for this article is retained by the author(s), with first publication rights granted to the journal.

This is an open-access article distributed under the terms and conditions of the Creative Commons Attribution license (http://creativecommons.org/licenses/by/3.0/). 